\title{
Glue or Onyx: A Guide to Choice - Tips and Techniques
}

\section{Enrico Maria Fumarola MD', Anna Maria lerardi MD², Filippo Piacentino MD³ and Gianpaolo Carrafiello MD PhD²}

\author{
'Diagnostic and Interventional Radiology Department, ASST Santi Paolo e Carlo, San Paolo Hospital, Milan, Italy \\ ²Unità Operativa di Radiologia, Fondazione I.R.C.C.S. Cà Granda Ospedale Maggiore Policlinico, Milan, Italy \\ ${ }^{3}$ Unit of Radiology, Ospedale di Circolo e Fondazione Macchi, University of Insubria, Varese, Italy
}

\begin{abstract}
Glue and Onyx are two liquid embolic agents. They have different characteristics and applications, so it is essential to know how to choose between them. The main aims of this article are to describe the principles and features of these embolic agents, to highlight the advantages and limitations of both the materials, and to provide optimal indications of each agent during endovascular arterial embolization procedures.
\end{abstract}

Keywords: Glue; NBCA; Onyx; Ethylene-vinyl Alcohol Copolymer; EVOH; Techniques; Embolization

Received: 3 February 2020; Accepted: 3 February 2020

\section{INTRODUCTION}

Onyx (Onyx Liquid Embolic System; ev3 Neurovascular, Irvine, CA, USA) and cyanoacrylate glue are two distinct embolic agents extensively used in peripheral vessel embolization.

The first report on the use of cyanoacrylate glue dates back to 1972 when it was successfully tested in an animal experiment on adult mongrel dogs to evaluate the possibility of its use as an embolic agent [1]. The first clinical series dates back to 1975 [2].

Onyx consists of an elastic polymer with an ethylenevinyl alcohol copolymer (EVOH) dissolved in an organic solvent, dimethyl sulfoxide (DMSO), with micronized tantalum powders to provide radiopacity.

The first animal experiment confirming the efficacy and safety of Onyx as an embolic agent was published in 1998 [3], and since then Onyx has been recognized as a valid tool for several embolization procedures. It was used initially in interventional neuroradiological procedures, particularly for the treatment of artero-venous malformations [4,5], but it subsequently became evident that Onyx could be used for the effective endovascular

\section{Corresponding author:}

Anna Maria lerardi, Diagnostic and Interventional Radiology Department, University of Milan, Via di Rudinì 8,

20142 Milan, Italy.

Email: amierardi@yahoo.it

(C) 2020 CC BY 4.0 - in cooperation with Depts. of Cardiothoracic/ Vascular Surgery, General Surgery and Anesthesia, Örebro University Hospital and Örebro University, Sweden management of peripheral bleeding and for the treatment of type-II endoleaks [6,7].

The main aims of this article are to describe the principles and features of these embolic agents, to highlight the advantages and limitations of both materials, and to provide optimal indications of each agent during endovascular arterial embolization procedures.

\section{MATERIALS DESCRIPTIONS}

\section{Onyx}

Onyx is a non-adhesive and non-absorbable permanent embolic agent that is injectable through DMSO-compatible catheters. It exists in liquid form and remains stable for as long as the liquid is saturated with a solvent.

Tantalum powder $(28 \%)$ is added to the $\mathrm{EVOH}$ polymer to make it radiopaque. When it comes into contact with water or blood, the material precipitates due to rapid diffusion of the DMSO solvent, with the formation of an elastic, soft, spongy and radiopaque cohesive cast inside the vessel lumen, which solidifies completely after about 10 minutes from solvent diffusion [8].

Onyx is available in two different viscosities: Onyx 18 (with 6\% EVOH) and Onyx 34 (with 8\% EVOH), and in two different formulas (1.5 and $6 \mathrm{ml})$. The lower the concentration of the copolymer, the less viscous the agent becomes, achieving more distal penetration when it is used for endovascular embolization. Its non-adhesivity allows for reliable delivery. Although there have been reports of Onyx's adhesion to the microcatheter tip, this risk is substantially lower than that posed by standard 


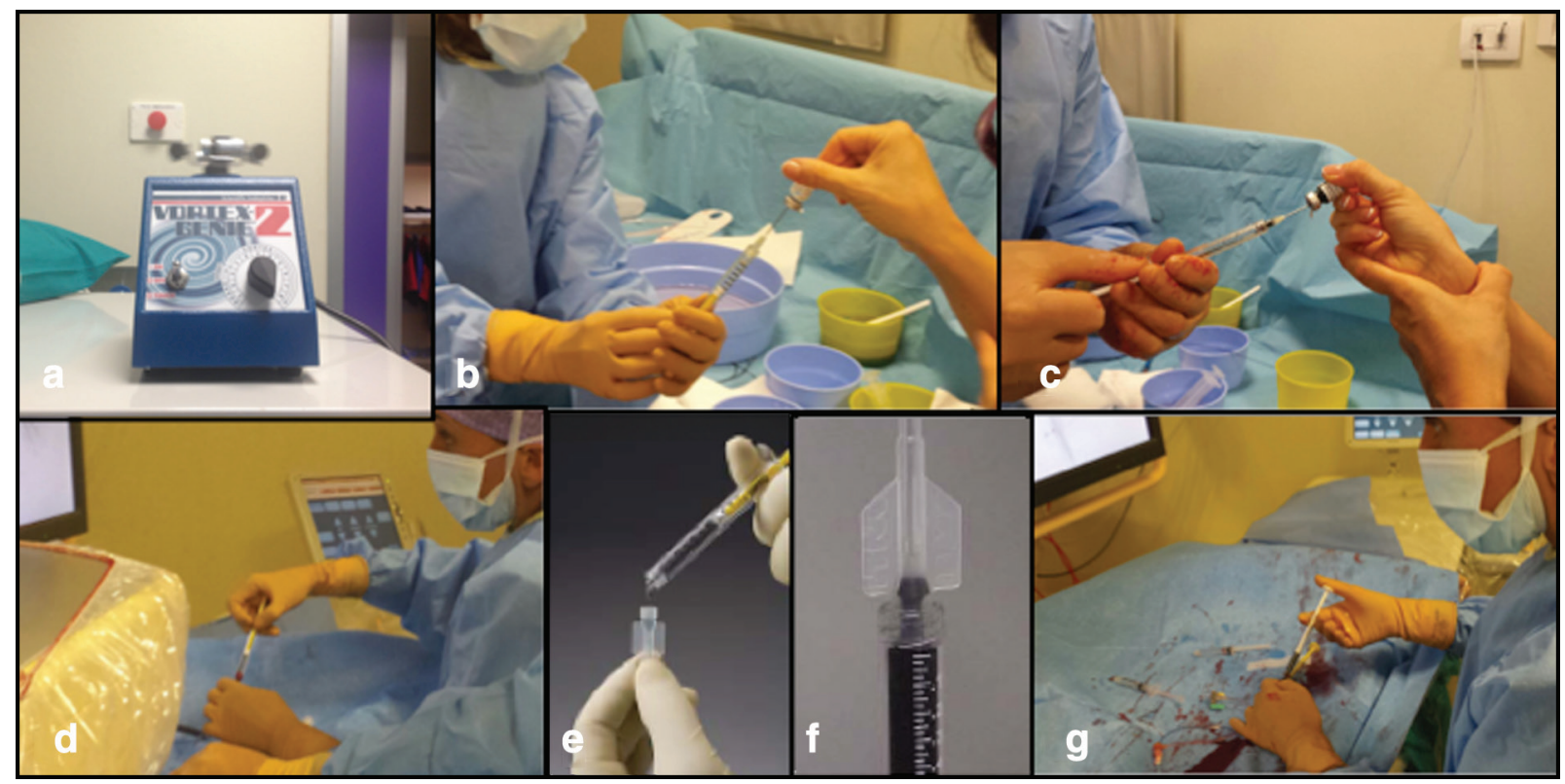

Figure 1 How to use Onyx. (a) Onyx Vial on a mixer for at least 20 minutes. (b, c) Fill the dedicated syringes with DMSO and Onyx respectively. (d) Flush a DMSO-compatible microcatheter with saline solution. (e) Then keep the microcatheter in a vertical position with the DMSO liquid surface at the hub of the microcatheter. $(\mathbf{f}, \mathbf{g})$ Connect the syringe containing Onyx to the microcatheter hub and start the injection.

glue; moreover, its viscosity helps to achieve optimal penetration of the embolic agent with slow injection [6].

A series of precise steps are necessary to assure expected delivery, avoiding complications such as excessive DMSO displacement into the blood stream, which can lead to severe, non-retractable pain related to endothelial necrosis.

How to use Onyx (Figure 1):

1. Place the Onyx vials on a mixer; the manufacturer suggests at least 20 minutes mixing time to obtain homogeneous distribution of the tantalum powders.

2. Flush a DMSO-compatible microcatheter with a saline solution.

3. Fill a 1-ml white syringe in the product package with Onyx through an 18 or $20 \mathrm{G}$ needle.

4. Aspirate the DMSO into a 1-ml yellow syringe in the product package and slowly fill the dead space of the microcatheter; then, keep the microcatheter in a vertical position with the DMSO liquid surface at the hub of the microcatheter to facilitate a "wet-towet" connection with the Onyx syringe, thereby avoiding contamination from air bubbles during the following step.

5. Connect the syringe containing Onyx to the microcatheter hub and start the injection (consider the dead space) under fluoroscopic guidance (the blank road map technique is preferred). It is important to inject the Onyx slowly, such as at a $0.16 \mathrm{ml} / \mathrm{min}$ $(0.25 \mathrm{ml} / 90 \mathrm{~s})$ injection rate, and not to exceed a rate of $0.3 \mathrm{ml} / \mathrm{min}$ to avoid vasospasm due to DMSO.

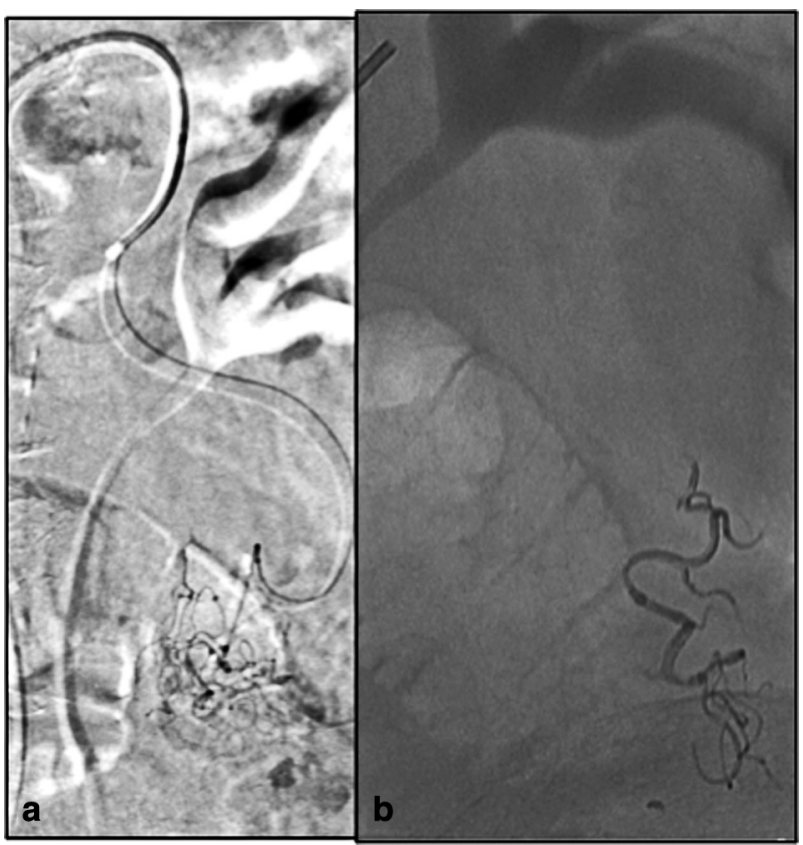

Figure 2 lleal bleeding. (a) Selective arteriography shows bleeding. (b) Successfully embolized with Onyx 18.

Cerebral arteriovenous malformations and distal fistulae have been the leading recognized indications of Onyx. Peripheral arteriovenous malformations meet the same criteria; accordingly, it was entirely reasonable to use Onyx to occlude nidus and afferent and efferent branches [6,9].

In peripheral applications, one of the first uses of Onyx was for the embolization of endoleaks, the rationale for 


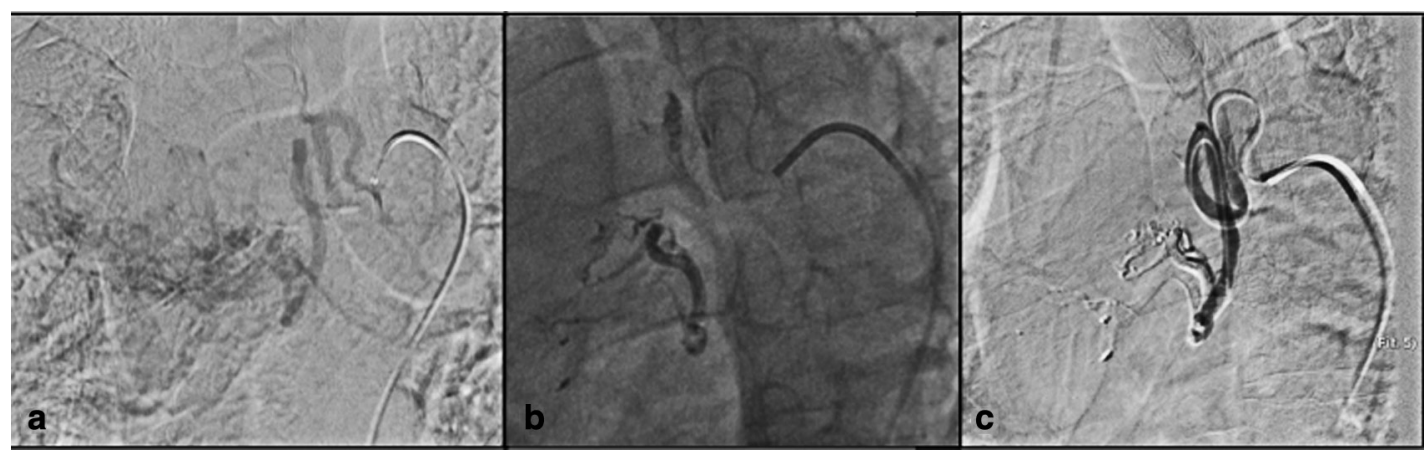

Figure 3 Bronchial artery embolization. (a) Massive hemoptysis in patient with hypertrophic right bronchial artery. (b, c) Embolized with Onyx 18 e 34.

which derived from the observation that recurrence is often caused by the incomplete filling of the sac. Onyx immediately came across as suitable for overcoming this limit, which is related to the use of other embolic agents $[6,9]$.

Post-traumatic and iatrogenic bleeding, especially pseudoaneurysms, may also be successfully embolized with Onyx [6]. Onyx can now be used in a wide range of procedures, including interventions in the venous district (esophageal varices, portal vein embolization), gastrointestinal bleeding embolization (Figure 2) and bronchial artery embolization (Figure 3a,b) [5,6,9-15].

Compared with cyanoacrylate glue, Onyx has some advantages: its viscous and non-adhesive properties, with slow and steady injection, make controlled delivery during embolization feasible; the controllability and standardized viscosities of the product, as well as its non-adhesive profile, subsequently allow for optimal penetration of the agent into the target vessels; and its cohesivity, allowing an efficient continuous casting inside the target vessels, which may require a smaller amount of embolic agent to achieve complete embolization. The use of a "plug and push" technique or balloon occlusion may offer a more controlled delivery method. Although the requirement of DMSO for Onyx may cause more chemical stimulation at the time of delivery, the non-adhesive and non-absorbable features of the Onyx polymer have a weaker inflammatory effect on the endothelium than cyanoacrylate glue once embolization is completed [6]. Although both Onyx and cyanoacrylate glue theoretically polymerize independently of the coagulation status of the patients, the superior casting profile of Onyx, in addition to its non-absorbability (as opposed to the absorbability of Lipiodol mixed with cyanoacrylate glue), may enable Onyx to provide more stable embolization in a setting of prolonged coagulopathy than cyanoacrylate glue. On the other hand, there are some drawbacks to Onyx: first, although it has only been described in rare cases, DMSO can cause severe vasospasm or endothelial injury [16], and the patient may experience severe pain, which can require anesthesiologic support to manage deep sedation $[6,9]$. The possibility of vasospasm with DMSO causing outflow stenosis or occlusion immediately before the injection of Onyx may also give rise to a concern about suboptimal proximal embolization, particularly in target vessels with progressive significant vasospasm (e.g. in a trauma patient). Second, the duration of the injection compared with glue can often be time consuming, which, according to some authors, may restrict use of the material to elective procedures only [17]. Finally, Onyx is more expensive than other commonly used embolic materials.

In conclusion, Onyx may be considered a useful, safe, and effective liquid embolic tool with a unique profile in the management of several conditions. Even though a non-negligible learning curve to handle it appropriately is required, it has become a powerful weapon for interventional radiologists.

\section{Glue}

Cyanoacrylate glue is the most commonly used liquid adhesive for endovascular procedures in interventional radiology [18]. It is composed of liquid alkyl-2-cyanoacrylate monomers that polymerize on contact with ionic materials such as blood, water, or endothelium [19]. Dextrose $5 \%$ in water (D5W), which is non-ionic, is used to flush the catheter before and often after administration of the glue to prevent its polymerization within the catheter [20].

The injection of cyanoacrylates into the blood stream leads to an acute inflammatory reaction of the vessel wall, which subsequently evolves into a chronic granulomatous foreign-body inflammatory reaction that leads to fibrosis within about 1 month [18].

By nature, the adhesive is not radiopaque and polymerizes in 1-2 s, which is not feasible for use in clinical practice. Modifications to address these issues include the addition of powdered metals, typically tantalum or tungsten, and iodized oils.

Iodized oils do not only make the glue radiopaque but also prolong the polymerization time.

Currently, in our clinical practice, we use Glubran2 (GEM Srl, Viareggio, Italy), which consists of a mix of 


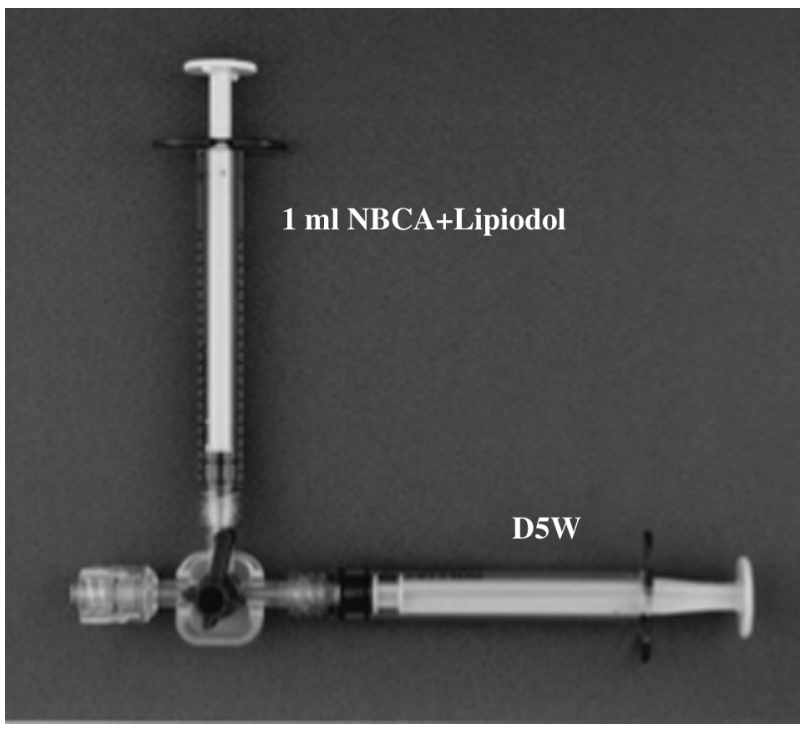

Figure 4 Two syringes (one with NBCA + Lipiodol, the other with dextrose $5 \%$ in water (D5W)), connected, ready to start embolization.

two monomers, $N$-butyl-2-cyanoacrylate (NBCA) and metacryloxysulfolane (MS), which are added to a Lipiodol solution (Guerbet, Roissy, France) to produce iodized oil emulsion in ratios ranging from 1:1 to 1:4.

A high NBCA-MS/Lipiodol ratio (1:1, 1:2), produced by adding a smaller amount of Lipiodol, offers quicker polymerization that restricts the time to inject the glue mixture through the catheter before it has to be removed due to possible adhesion of the injected glue to the catheter tip after polymerization. On the other hand, a low NBCA-MS/Lipiodol ratio (1:3, 1:4) allows more time for injection while increasing the risk of distal embolization [21].

Blood-flow control should be considered when NBCA migration is expected in high-flow lesions. Flow stagnation via the use of balloon catheters or wedge catheters, or the combined use of vascular embolization and metallic coils or other materials, and temporary vasoconstriction via the local injection of diluted epinephrine, have been reported in such situations [22].

How to use cyanoacrylate glue:

1. Use a microcatheter using a coaxial technique advancing the catheter into a safe position to avoid reflux.

2. Perform a diagnostic angiography beforehand to confirm the exact position of your target and to assess flow dynamics.

3. Flush the microcatheter with D5W.

4. Inject the cyanoacrylate + Lipiodol; operators should closely monitor the progression of the glue during injection with the use of fluoroscopy, digital subtraction angiography, and road-mapping (Figure 4) [22].
5. Flush the microcatheter immediately with D5W, or remove the microcatheter with negative pressure on the syringe, to avoid inadvertent adherence to the surrounding vascular tissue, according to the specific glue-embolization technique used.

The continuous column technique: The glue mixture is injected slowly but continuously to allow it to form a cast inside the lumen, which allows controlled NBCA delivery, avoiding dangerous refluxes [22]. As soon as the injection is complete, the microcatheter must be pulled out swiftly in one rapid motion and taken out of the guiding catheter. Upon withdrawal, the microcatheter must be immediately flushed with D5W, for re-use in order to reduce costs [23].

The "drop-by-drop" technique [22]: The tip of the microcatheter has to be positioned as near as possible to the target lesion in order to prevent the administration of an excess amount of glue. NBCA is then injected "drop-by-drop" in order to maximize the dose. The injection should be stopped when the blood flow stops, or if NBCA lies alongside the catheter tip, in order to avoid severe symptoms or non-target embolization.

The "sandwich" technique: This is a method for alternate injections of glue and D5W [24]. The use of a three-way stopcock to allow faster flushing of the microcatheter with $\mathrm{D} 5 \mathrm{~W}$ between pulses of glue injection is highly recommended. Small aliquots of glue not exceeding the dead volume of the microcatheter $(0.5-0.7 \mathrm{ml}$ in most cases) should be injected followed by $\mathrm{D} 5 \mathrm{~W}$ to clear the catheter and drive the glue into the target arteries. Additional aliquots of glue followed by D5W are then injected to achieve optimal embolization.

In 2014, the Japanese Society of Interventional Radiology, acting in accordance with the Committee of Practice Guidelines of the Japanese Society of Interventional Radiology, developed a document to describe the current consensus regarding the use of NBCA for vascular embolization [22].

Cyanoacrylate is a highly adaptable adhesive and can be used in a wide variety of endovascular procedures: peripheral and gastrointestinal embolization (even in the presence of coagulopathies; Figure 5), arteriovenous vascular malformations (Figure 6), type-II endoleak embolization of aortic aneurysms, treatment of venous district conditions (esophageal varices, portal vein embolization), oncological treatment (both palliative and preoperative), and bronchial artery embolization for the treatment of hemoptysis [12,15,18,20,21,25-29].

There are advantages to the use of cyanoacrylate beyond its broad usability: it polymerizes in only a few seconds to achieve prompt hemostasis; and the product 


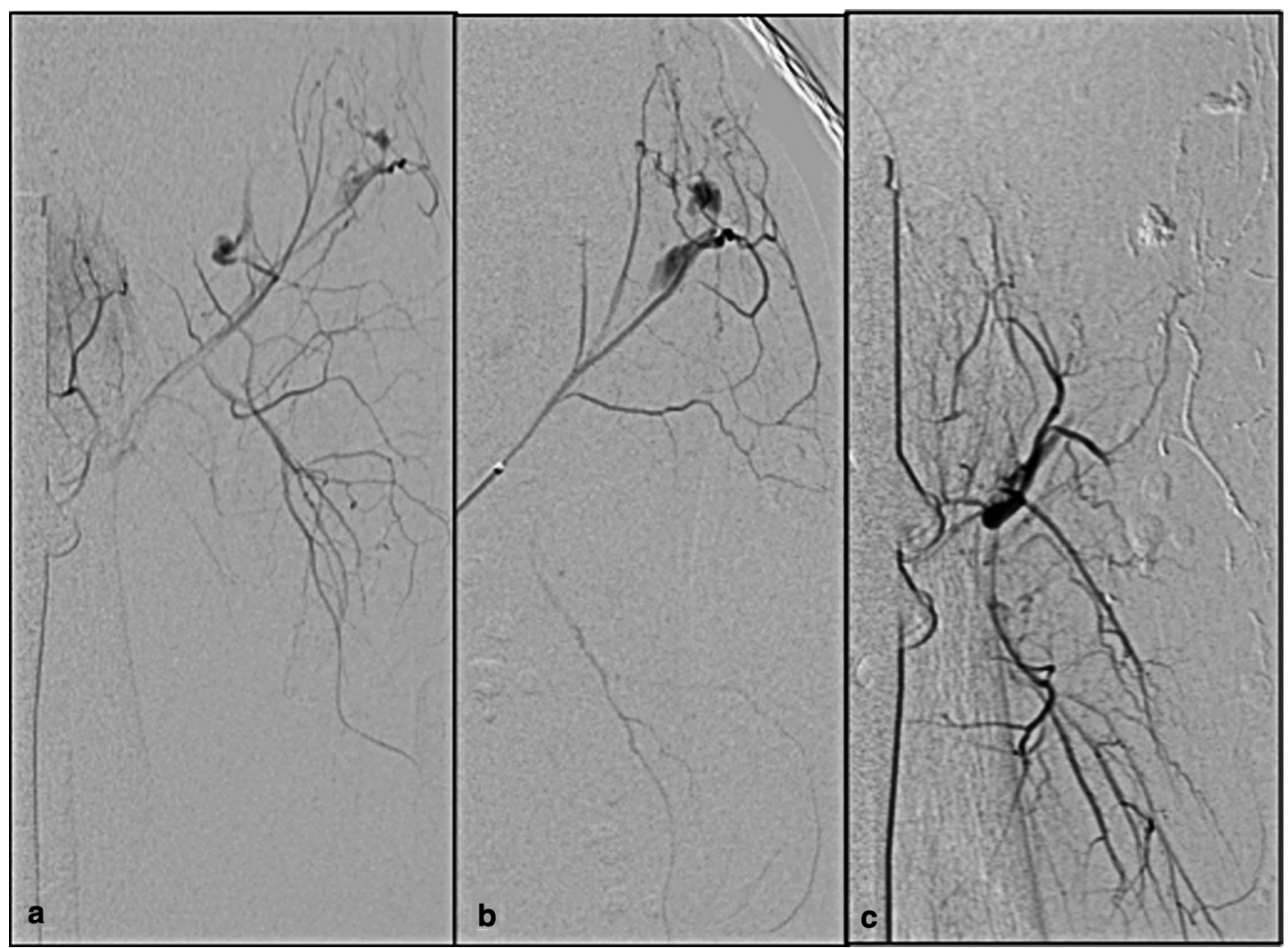

Figure 5 Peripheral embolization. (a, b) Bleeding of the lateral circumflex artery. (c) Embolized with glue (1:3).

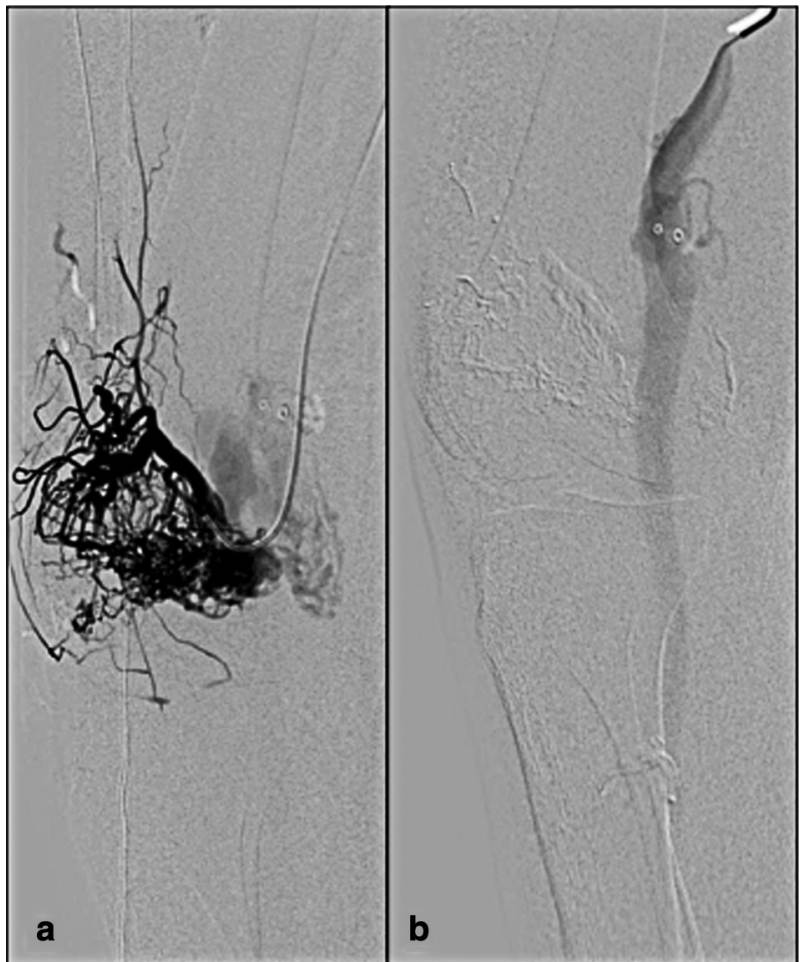

Figure 6 Arteriovenous vascular malformations. (a) Selective angiography of a voluminous artero-venous malformation. (b) Embolized with glue (1:3). is almost ready-to-use in a few simple steps. In fact, it can be quickly administered, only requiring dilution with Lipiodol before its injection, which is a crucial feature especially when dealing with a hemodynamically unstable patient [21]. Also, cyanoacrylates are effective for hemostasis because of their independent polymerization mechanism even in the setting of a coagulation disorder, which is often present in arterial bleeding because of anticoagulant medication or is induced by the hemorrhagic shock itself. Further, it allows for the embolization of small or tortuous vessels due to its liquidity. Finally, embolization with glue is very cost-effective in most countries in the world; $1 \mathrm{ml}$ is comparable in cost with a single conventional pushing coil [21].

There are also some drawbacks: cyanoacrylates are difficult to control during injection, and require high expertise due to serious potential complications, such as catheter-tip trapping or fragmentation and non-target embolization, depending on the cyanoacrylate/iodized oil ratio; the latter, in particular, needs specific attention due to its potential dramatic consequences. In this sense, information about flow dynamics and anatomical orientations provided by the test injection immediately before the embolization can be very helpful in predicting the appropriate injection rate to avoid refluxes. Lipiodol flow is slower than the flow of contrast medium in the normal setting owing to its higher viscosity. Moreover, operators have to consider that Lipiodol 
viscosity varies with temperature, and is especially low at high temperatures [22,30].

In conclusion, the use of glues in interventional radiology as an embolic agent is effective, safe, and inexpensive, and may be particularly useful in settings of hemodynamic instability, coagulopathy, vessels with underlying significant vasospasm, and small or tortuous vessels. Nevertheless, the glues require careful preprocedural assessment, attention to technical details, and a good amount of experience.

\section{DISCUSSION}

Both Onyx and cyanoacrylate glue have the great advantage of polymerizing independently of any underlying coagulopathy or low platelet count compared with mechanical devices, such as coils, which rely on patients' coagulation mechanisms for complete hemostasis [31]. Both agents can also be used for a wide range of interventions and in different areas.

To our knowledge, there are no prospective studies that compare Onyx and glues in terms of efficacy or safety in an extracranial setting.

What emerges from the literature is that Onyx's physical properties allow controlled and precise delivery of the embolic liquid so that it reaches specific distal arterial branches. Also, Onyx works equally well with or without flow, particularly with the "plug and push" technique, which might be an advantage in cases where non-occlusive vasospasm is present despite some concern about worsening underlying vasospasm with DSMO injections. By contrast, decent forward flow is necessary for cyanoacrylate glue to be appropriately delivered [31].

A great concern with cyanoacrylate glue is the difficulty in achieving controlled delivery, which is inevitably associated with the risk of distal migration or reflux to the proximal anatomy that will cause non-target embolization. Risks related to microcatheter entrapment, particularly with a high concentration of cyanoacrylate, are also a major concern [31].

Economic considerations, however, greatly favor the use of glue in the majority of countries. As already mentioned, $1 \mathrm{ml}$ of glue is comparable in cost with a single conventional pushing coil and is sufficient for successful treatment in the majority of cases [21]. By contrast, Onyx is far more expensive, which can limit its availability in complex procedures in more noble districts that you have to handle with care (brain, gastrointestines), where the risks of non-target embolization outweigh the benefits of using cyanoacrylate glue.

\section{Ethics Statement}

(1) All the authors mentioned in the manuscript have agreed to authorship, read and approved the manuscript, and given consent for submission and subsequent publication of the manuscript.
(2) The authors declare that they have read and abided by the JEVTM statement of ethical standards including rules of informed consent and ethical committee approval as stated in the article.

\section{Conflicts of Interest}

The authors declare that they have no conflicts of interest.

\section{Funding}

The authors received no financial support for the research, authorship, and/or publication of this article.

\section{REFERENCES}

[1] Zanetti PH, Sherman FE. Experimental evaluation of a tissue adhesive as an agent for the treatment of aneurysms and arteriovenous anomalies. J Neurosurg. 1972; 36:72-9.

[2] Dotter CT, Goldman ML, Rösch J. Instant Selective Arterial Occlusion with Isobutyl 2-Cyanoacrylate. Radiology. 1975;114:227.

[3] Murayama Y, Viñuela F, Ulhoa A, et al. Nonadhesive liquid embolic agent for cerebral arteriovenous malformations: preliminary histopathological studies in swine rete mirabile. Neurosurgery. 1998;43:1164-72.

[4] Molyneux AJ, Coley SC. Embolization of spinal cord arteriovenous malformations with an ethylene vinyl alcohol copolymer dissolved in dimethyl sulfoxide (Onyx liquid embolic system): report of two cases. J Neurosurg. 2000;93:304-8.

[5] Gobin YP, Duckwiler GR, Vinters H V, Viñuela F, Jahan R, Murayama Y. Embolization of arteriovenous malformations with Onyx: clinicopathological experience in 23 patients. Neurosurgery. 2001;48:984-95; discussion 995-7.

[6] Ierardi AM, Femia M, Petrillo M, Angileri SA, Hörer T, Carrafiello G. Onyx liquid embolic system (LES): an underestimated tool in the management of peripheral bleedings. J Endovasc Resusc Trauma Manag. 2018;2:49-56.

[7] Martin ML, Dolmatch BL, Fry PD, Machan LS. Treatment of type II endoleaks with Onyx. J Vasc Interv Radiol. 2001;12:629-32.

[8] Vanninen RL, Manninen I. Onyx, a new liquid embolic material for peripheral interventions: preliminary experience in aneurysm, pseudoaneurysm, and pulmonary arteriovenous malformation embolization. Cardiovasc Intervent Radiol. 2007;30:196-200.

[9] Kolber MK, Shukla PA, Kumar A, Silberzweig JE. Ethylene vinyl alcohol copolymer (Onyx) Embolization for acute hemorrhage: a systematic review of peripheral applications. J Vasc Interv Radiol. 2015;26:809-15.

[10] Khaja MS, Park AW, Swee W, et al. Treatment of type II endoleak using Onyx with long-term imaging follow-up. Cardiovasc Intervent Radiol. 2014;37:613-22.

[11] Marcelin C, Le Bras Y, Petitpierre F, et al. Embolization for persistent type IA endoleaks after chimney endovascular aneurysm repair with Onyx ${ }^{\circledast}$. Diagn Interv Imaging. 2017;98:849-55. 
[12] Rostambeigi N, Shrestha P, Dunn TB, et al. Recurrent ectopic variceal bleed after pancreas transplantation with no portal hypertension: case report and outcomes of endovascular onyx embolization. Vasc Endovascular Surg. 2019;53:415-9.

[13] Gabrielli R, Bafile G, Filauri P, D'Elia M. Symptomatic pancreaticoduodenal artery aneurysm in a patient with prior aortic surgery and celiac trunk obstruction: case report. Ann Vasc Surg. 2019;61:471.e1-471.e2.

[14] Saeed Kilani M, Izaaryene J, Cohen F, et al. Ethylene vinyl alcohol copolymer (Onyx®) in peripheral interventional radiology: indications, advantages and limitations. Diagn Interv Imaging. 2015;96:319-26.

[15] Bommart S, Bourdin A, Giroux MF, et al. Transarterial ethylene vinyl alcohol copolymer visualization and penetration after embolization of life-threatening hemoptysis: technical and clinical outcomes. Cardiovasc Intervent Radiol. 2012;35:668-75.

[16] Siekmann R. Basics and principles in the application of Onyx LD liquid embolic system in the endovascular treatment of cerebral arteriovenous malformations. Interv Neuroradiol. 2005;11:131-40.

[17] Loffroy R, Rao P, Ota S, De Lin M, Kwak BK, Geschwind JF. Embolization of acute nonvariceal upper gastrointestinal hemorrhage resistant to endoscopic treatment: results and predictors of recurrent bleeding. Cardiovasc Intervent Radiol. 2010;33:1088-100.

[18] Pollak JS, White J. The use of cyanoacrylate adhesives in peripheral embolization. J Vasc Interv Radiol. 2001;12: 907-13.

[19] Cromwell LD, Kerber CW. Modification of cyanoacrylate for therapeutic embolization: preliminary experience. Am J Roentgenol. 1979;132:799-801.

[20] Madhusudhan KS, Venkatesh HA, Gamanagatti S, Garg $\mathrm{P}$, Srivastava DN. Interventional radiology in the management of visceral artery pseudoaneurysms: a review of techniques and embolic materials. Korean J Radiol. 2016; 17:351-63.

[21] Abdulmalak G, Chevallier O, Falvo N, et al. Safety and efficacy of transcatheter embolization with Glubran ${ }^{\circledR} 2$ cyanoacrylate glue for acute arterial bleeding: a singlecenter experience with 104 patients. Abdom Radiol. 2018; 43:723-33.
[22] Takeuchi Y, Morishita H, Sato Y, et al. Guidelines for the use of NBCA in vascular embolization devised by the Committee of Practice Guidelines of the Japanese Society of Interventional Radiology (CGJSIR), 2012 edition. Jpn J Radiol. 2014;32:500-17.

[23] Baltacıŏlu F, Çimşit NÇ, Bostanci K, Yüksel M, Kodalli $\mathrm{N}$. Transarterial microcatheter glue embolization of the bronchial artery for life-threatening hemoptysis: technical and clinical results. Eur J Radiol. 2010;73:380-4.

[24] Razavi MK, Murphy K. Embolization of bronchial arteries with N-butyl cyanoacrylate for management of massive hemoptysis: a technical review. Tech Vasc Interv Radiol. 2007;10:276-82.

[25] Raffi L, Simonetti L, Cenni P, Leonardi M. Use of Glubran 2 acrylic glue in interventional neuroradiology. Neuroradiology. 2007;49:829-36.

[26] Torikai H, Hasegawa I, Jinzaki M, Narimatsu Y. Preliminary experience of endovascular embolization using N-butyl cyanoacrylate for hemoptysis due to infectious pulmonary artery pseudoaneurysms via systemic arterial approach. J Vasc Interv Radiol. 2017;28:1438-42. e1.

[27] Goral V, Yilmaz N. Current approaches to the treatment of gastric varices: glue, coil application, TIPS, and BRTO. Medicina (B Aires). 2019;55:335.

[28] Mukund A, Mondal A, Patidar Y, Kumar S. Safety and outcomes of pre-operative portal vein embolization using N-butyl cyanoacrylate (Glue) in hepatobiliary malignancies: A single center retrospective analysis. Indian J Radiol Imaging. 2019;29:40.

[29] Guziński M, Kurcz J, Tupikowski K, Antosz E, Słowik P, Garcarek J. The role of transarterial embolization in the treatment of renal tumors. Adv Clin Exp Med. 2015;24: 837-43.

[30] Li YJ, Barthès-Biesel D, Salsac AV. Polymerization kinetics of N-butyl cyanoacrylate glues used for vascular embolization. J Mech Behav Biomed Mater. 2017;69: 307-17.

[31] Urbano J, Manuel Cabrera J, Franco A, Alonso-Burgos A. Selective arterial embolization with ethylene-vinyl alcohol copolymer for control of massive lower gastrointestinal bleeding: Feasibility and initial experience. J Vasc Interv Radiol. 2014;25:839-46. 